\title{
Escherichia coli mar and acrAB Mutants Display No Tolerance to Simple Alcohols
}

\author{
Jonas Ankarloo ${ }^{1}$, Susanne Wikman ${ }^{1}$ and Ian A. Nicholls ${ }^{1,2, *}$
}

1 Bioorganic \& Biophysical Chemistry Laboratory, School of Natural Sciences, Linnaeus University, SE-391 82 Kalmar, Sweden; E-Mails: jonas.ankarloo@lnu.se (J.A.); susanne.wikman@lnu.se (S.W.)

2 Department of Biochemistry \& Organic Chemistry, Uppsala University, Box 576, SE-751 23 Uppsala, Sweden

* Authors to whom correspondence should be addressed; E-Mail: ian.nicholls@lnu.se; Tel.: +46-480-446-258; Fax: +46-480-446-244.

Received: 1 February 2010; in revised form: 28 March 2010 / Accepted: 29 March 2020 / Published: 31 March 2010

\begin{abstract}
The inducible Mar phenotype of Escherichia coli is associated with increased tolerance to multiple hydrophobic antibiotics as well as some highly hydrophobic organic solvents such as cyclohexane, mediated mainly through the AcrAB/TolC efflux system. The influence of water miscible alcohols ethanol and 1-propanol on a Mar constitutive mutant and a mar deletion mutant of E. coli K-12, as well as the corresponding strains carrying the additional $\operatorname{acr} A B$ deletion, was investigated. In contrast to hydrophobic solvents, all strains were killed in exponential phase by 1-propanol and ethanol at rates comparable to the parent strain. Thus, the Mar phenotype does not protect E. coli from killing by these more polar solvents. Surprisingly, AcrAB does not contribute to an increased alcohol tolerance. In addition, sodium salicylate, at concentrations known to induce the mar operon, was unable to increase 1-propanol or ethanol tolerance. Rather, the toxicity of both solvents was increased in the presence of sodium salicylate. Collectively, the results imply that the resilience of $E$. coli to water miscible alcohols, in contrast to more hydrophobic solvents, does not depend upon the AcrAB/TolC efflux system, and suggests a lower limit for substrate molecular size and functionality. Implications for the application of microbiological systems in environments containing high contents of water miscible organic solvents, e.g., phage display screening, are discussed.
\end{abstract}


Keywords: solvent tolerance; salicylate; ethanol; 1-propanol; mar regulon; hydrophobicity; solvent

\section{Introduction}

Many, but not all, organic solvents are toxic to microorganisms. The toxicity of organic solvents in two-phase water-solvent systems correlates inversely with the $\log P_{\text {ow }}$ (the partition coefficient between $n$-octanol and water) of the solvent; in the range between 1 and 5 . The ability of solvent molecules to partition into the water phase and into the cell membrane(s) is of great importance for toxicity as highly non-polar solvents ( $\log P_{\text {ow }}$ above 5) show no adverse effect on the metabolic activity in several bacterial species [1]. For readily water-miscible organic solvents such as short-chain alcohols (e.g., methanol, ethanol and 1-propanol), toxicity correlates directly with hydrophobicity [2,3].

The cytoplasmic membrane is the main site of action for organic solvents. Using 1,2,3,4-dihydronaphthalene (tetralin), Sikkema and coworkers [4] demonstrated a decrease in membrane potential $(\Delta \mathrm{p})$, chiefly dependent on a lowered internal $\mathrm{pH}$ and transmembrane $\Delta \mathrm{pH}$, in cytochrome $\mathrm{C}$ oxidase-reconstituted liposomes. This effect was ascribed to an increased $\mathrm{H}^{+}-$ permeability of the membrane, and was concomitant with growth arrest in similarly treated sensitive bacteria. Furthermore, cyclic hydrocarbons were shown to dissipate both the $\mathrm{pH}$ gradient and the electrochemical potential [5]. Ethanol has been shown to affect the proton motive force [6], and to increase leakage of metabolites from cells [7].

Inducible multi-antibiotic-, disinfectant- and oxidative stress agent-tolerance regulons have been identified in several bacterial species [8-10]. The E. coli mar operon contains a promotor under negative control of the MarR repressor, which is co-transcribed with the marA gene [11]. This gene encodes a transcriptional activator affecting the expression of some 60 chromosomal genes [12] including the up-regulation of the AcrAB plasma membrane efflux transporter and TolC outer membrane channel. As a result, structurally unrelated hydrophobic or amhiphilic compounds such as $\beta$-lactams, tetracycline, chloramphenicol, fluoroquinolones $[13,14]$ as well as bile salts [15] and some disinfectants [16,17] may be pumped across both membranes. Null mutations in marR cause the overexpression of marA and constitutive multi-antibiotic tolerance (the Mar phenotype). In addition, the mar operon is inducible in wild-type strains, e.g., by low levels of tetracycline or chloramphenicol, but not ampicillin [18]. In the absence of the AcrAB pump, marR mutations fail to display the Mar phenotype [19].

Salicylate has been shown to induce the mar operon by directly binding to, and inactivating MarR [20,21]. The redundancy in hydrophobic substance-extruding pumps is evident in that salicylate weakly increases antibiotic tolerance (excluding aminoglycosides) in marA-deleted E. coli [22]. Indeed, salicylate has been shown to relieve emrRAB repression by binding to EmrR [23].

The previous isolation of cyclohexane-tolerant E. coli K-12 mutants that showed multi-antibiotic tolerance [24] raised the question as to whether there was a common denominator between solventand antibiotic tolerance in E. coli. Indeed, one such mutant proved to be marR [25], suggesting a 
common stress-dependent resistance mechanism. Clinical isolates of E. coli exhibiting fluoroquinolone resistance, have shown an increased proportion of cyclohexane-tolerant strains [26]. Moreover, plasmid-mediated overexpression of marA has been shown to increase tolerance to both cyclohexane and hydrophobic antibiotics [25]. The objective of the present study was to establish if Mar activation could also increase tolerance to water-miscible solvents (ethanol, 1-propanol).

\section{Results and Discussion}

\subsection{Exponential-phase Killing by Ethanol or 1-Propanol Is Unaffected by the mar Phenotype}

At $10.0 \%(\mathrm{w} / \mathrm{w})$ and $4.05 \%(\mathrm{w} / \mathrm{w})$, respectively, ethanol and 1-propanol killed the mar as well as the parent E. coli strains exponentially, with single-hit kinetics (Figure 1). Despite the rapid loss of viability, optical density of the cultures increased slightly during the first 30-60 min after alcohol addition (not shown), which is indicative of a growth in the size of viable bacteria, with no apparent osmotic lysis.

No statistically significant differences between any strains in 30-, 60- or 90-minute survival fractions could be detected, neither in the ethanol-treated (Figure 1a), nor in the 1-propanol-treated (Figure 1b) cultures. The Mar phenotype expressed in E. coli AG112 does not protect this strain against ethanol- or 1-propanol-mediated killing, compared to the parent strain. The deletion of marA in E. coli AG100/Kan does not render this strain more susceptible to ethanol or 1-propanol, thus mar induction may not significantly contribute to tolerance towards these alcohols.

Figure 1. Relative survival of E. coli AG100 (black diamonds), AG112 marR (open squares) and $A G 100 / \operatorname{Kan} \triangle(\operatorname{marCORAB})$ (open circles) after exposure to (a) $10.0 \%(\mathrm{w} / \mathrm{w})$ ethanol, or (b) $4.05 \%$ (w/w) 1-propanol. Cells were cultured in modified LB (see Materials and Methods section) until late exponential phase before the addition of solvent and further incubation. Error bars represent $95 \%$ confidence intervals based upon 8 replicates.
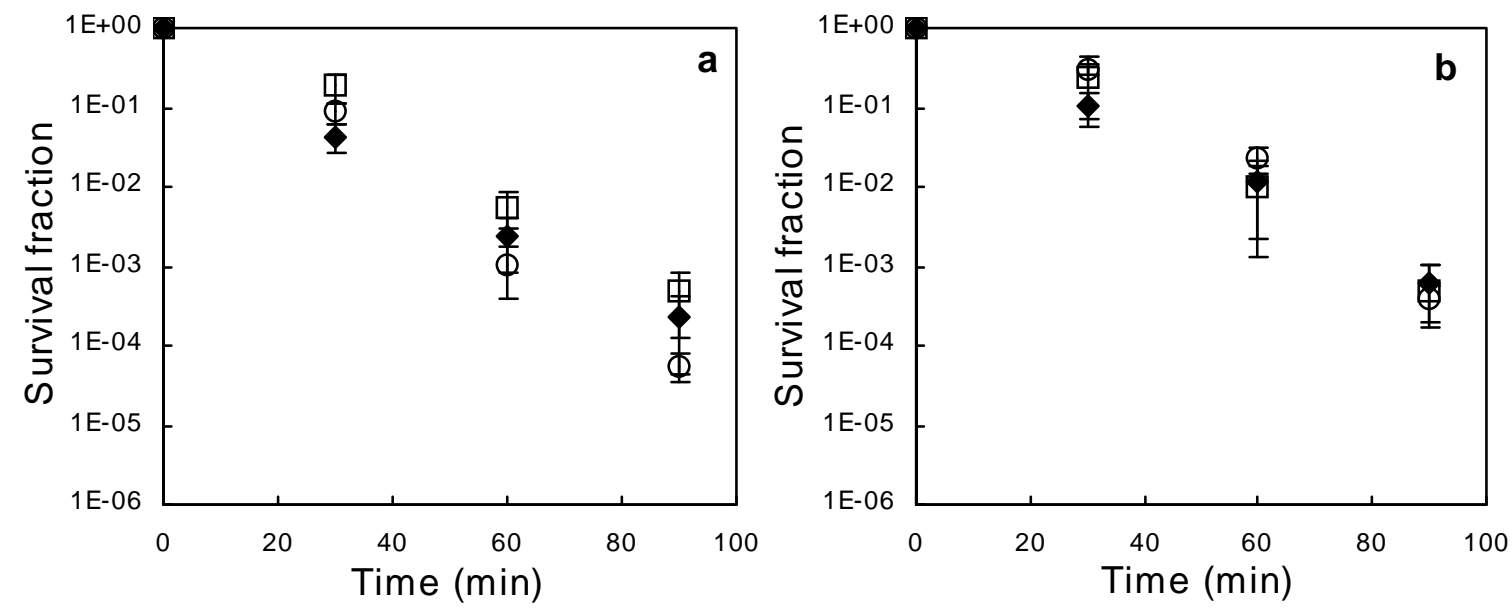

Growing E. coli are generally more solvent-sensitive than non-growing cells [2], therefore cultures in mid- to late exponential phase were chosen for subsequent solvent addition. In the stationary phase, solvent tolerance mechanisms other than Mar would take effect. Stationary-phase-dependent 
expression of the alternative sigma factor RpoS up-regulates $u s p B$, which is required for stationaryphase resistance to ethanol [30].

Growth is but one indicator of the physiological status of bacterial cells. E. coli generation times increase in the presence of low concentrations of ethanol [31], presumably through the diminished cellular energy status. At 5\% (v/v), ethanol causes growth arrest after one mass doubling with incomplete septation, also indicating an effect on cell wall synthesis [31]. This growth arrest could be alleviated by dilution with fresh medium. In contrast, the ethanol- and 1-propanol effect observed here is irreversible, killing the cells. In minimal medium, $5 \%(\mathrm{v} / \mathrm{v})$ of ethanol has been shown to cause cellular lysis in the absence, but not in the presence, of $0.1 \mathrm{M} \mathrm{NaCl}$. Peptidoglycan cross-linking was also shown to decrease [2]. It was proposed that peptidoglycan cross-linking enzymes may be inhibited because ethanol generally weakens hydrophobic interactions within the periplasm as well as the membrane interior, and that this may be overcome by $\mathrm{NaCl}$, as well as other antichaotropic salts. Similar lysis has been reported in E. coli grown in Luria-Bertani broth containing up to $5 \%(\mathrm{v} / \mathrm{v})$ of ethanol [32]. That no gross lysis ensued in our case may be surprising, especially since $\mathrm{NaCl}$ was omitted from the growth medium. However, actively growing cells, simultaneously synthesizing poorly cross-linked peptidoglycan and displaying autolysin activity, would be expected to readily lyse. Rapid cellular inactivation due to other mechanisms, at the relatively high alcohol concentrations used here may hence mask any impairment of cell wall synthesis, or autolysins may simply be just as rapidly inhibited.

\subsection{Salicylate Increases the Killing Rate of Ethanol and 1-Propanol, Irrespective of mar Induction}

E. coli AG100/Kan is a mar deletion mutant lacking most of the mar operon including the mar repressor and Mar activator genes. Salicylate can elicit no MarA-dependent solvent tolerance in this strain, but hypothetically so in the parent strain AG100 as cyclohexane tolerance was earlier shown to increase by salicylate addition (or marA overexpression) in E. coli W3110; not carrying any mar mutation [25]. Addition of 10.0\% ethanol to E. coli AG100 and AG100/Kan grown in the presence of $2 \mathrm{mM}$ sodium salicylate results in an increased killing rate, compared to AG100 grown without salicylate (Figure 2a). Similarly, salicylate increases the killing rate of 4.05\% 1-propanol in both strains (Figure 2b). The 60- and 90-minute survival fractions of salicylate/solvent-exposed AG100 as well as AG100/Kan differ significantly from non-salicylate treated AG100, for both solvents. In contrast, no statistically significant differences between AG100 and AG100/Kan survival rates in the presence of salicylate can be shown. Therefore, salicylate $(2 \mathrm{mM})$ significantly enhances ethanol- and 1-propanol mediated killing of E. coli K-12, irrespective of any salicylate-mediated Mar induction. This further strenghtens our view that the Mar phenotype cannot protect E. coli against lower primary alcohol (ethanol, 1-propanol) mediated killing.

Interestingly, synergism between a weak acid and ethanol in killing of $E$. coli has been described before [33]. $50 \mathrm{mM}$ of lactate in combination with $5 \%(\mathrm{v} / \mathrm{v})$ ethanol enhanced killing of $E$. coli O157:H7 about four log units in the exponential phase (first hour after addition), compared to ethanol only. It was also found that both lactate and ethanol independently lowered the cytoplasmic $\mathrm{pH}$ of the cells. We have not investigated cellular $\mathrm{pH}$, but salicylate at $2.5 \mathrm{mM}$, close to the concentration used here, has been shown to increase bacterial sensitivity towards aminoglycosides [34,35]. This has been 
proposed to partly result from intracellular deprotonation following salicylic acid diffusion across the cytoplasmic membrane [36,37].

Figure 2. Relative survival of E. coli AG100 (open squares) and AG100/Kan $\Delta(\operatorname{marCORAB})$ (open circles) grown in the presence of sodium salicylate, and $E$. coli AG100 grown in LB without salicylate (black diamonds) after subsequent exposure to (a) $10.0 \%(\mathrm{w} / \mathrm{w})$ ethanol, or (b) 4.05\% (w/w) 1-propanol. Cells were cultured in modified LB (see Materials and Methods section) containing $2 \mathrm{mM}$ sodium salicylate until late exponential phase before the addition of solvent and further incubation. Error bars represent $95 \%$ confidence intervals based upon 8 replicates.
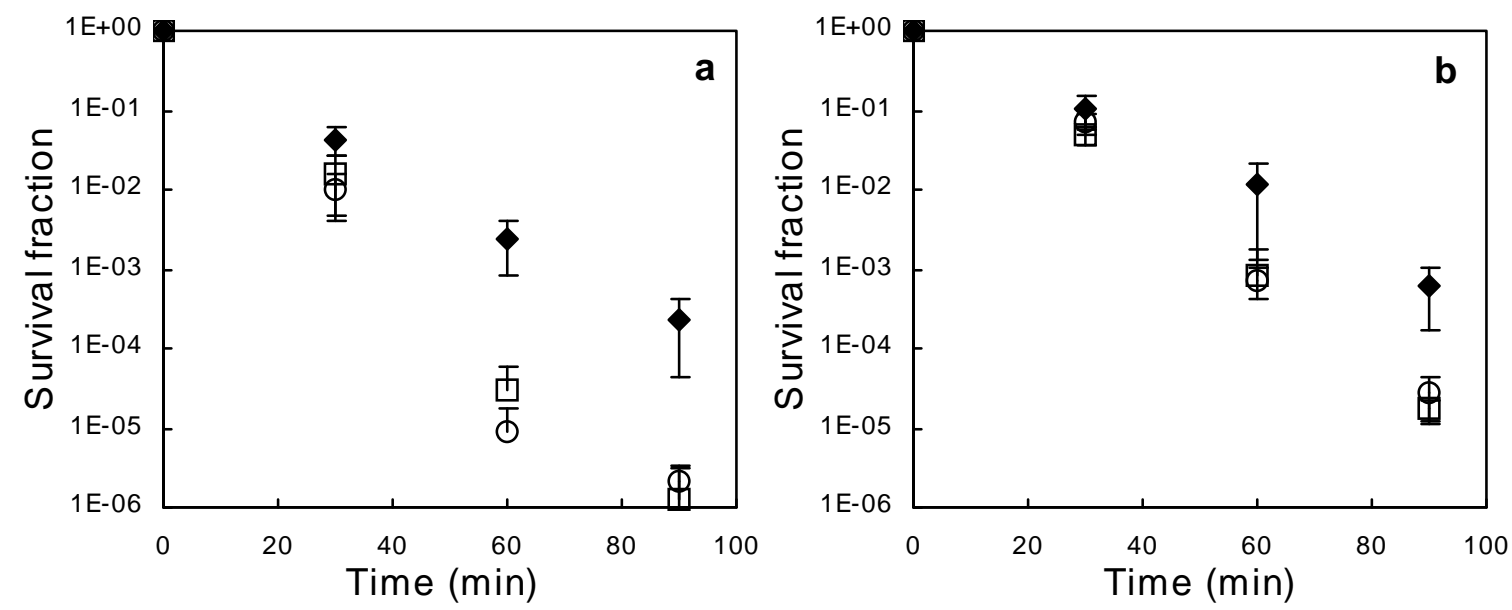

\subsection{Deletion of acrAB Does Not Increase Sensitivity to Ethanol or 1-Propanol}

Ma and coworkers [14] have described the induction of acr $A B$ transcription by $4 \%$ ethanol, even in a $\Delta$ mar strain; thus independently of the Mar response. In this study they did establish whether ethanol was a substrate of the AcrAB pump. AcrAB and TolC are both necessary to extrude solvents with a $P_{\text {ow }}$ greater than 3.4 [38]. We measured the killing rate of ethanol and 1-propanol in $\triangle($ acr $A B)$ as well as $\triangle(a c r A B)$ marR strains (Figure 3). Again, no statistically significant differences between AG100, AG100A and AG102/K survival rates after ethanol or 1-propanol exposure could be shown. This suggests that the AcrAB efflux pump cannot pump ethanol or 1-propanol across the membrane.

In summary, the fact that salicylate similarly enhanced ethanol- and 1-propanol-mediated killing, and that a lower concentration of the more hydrophobic 1-propanol was required to reach a comparable killing rate, suggest a common, synergistic relationship between salicylate and the alcohols, as well as a common, general killing mechanism presumably based on the partitioning of solvent into the plasma membrane. Neither the Mar phenotype, a well-characterised multi-drug and organic solvent tolerance phenotype, nor any stress-dependent induction of Mar or its central component AcrAB could alleviate the effect of ethanol- and 1-propanol. Collectively, these results establish a lower limit in terms of size and functionality for substrates for the AcrAB pump, information which should prove valuable for understanding resistance mechanisms, and for the use of microorganisms in biotechnological processes involving organic solvents $[39,40]$. 
Figure 3. Relative survival of E. coli AG100 (black diamonds), AG100A $\triangle(\operatorname{acr} A B)$ (open squares) and $A G 102 \mathrm{~K}$ marR $\Delta(\operatorname{acr} A B)$ (open circles) after exposure to a) $10.0 \%(\mathrm{w} / \mathrm{w})$ ethanol, or b) $4.05 \%$ (w/w) 1-propanol. Cells were cultured in modified LB (see Materials and Methods section) until late exponential phase before the addition of solvent and further incubation. Error bars represent $95 \%$ confidence intervals based upon 8 replicates.
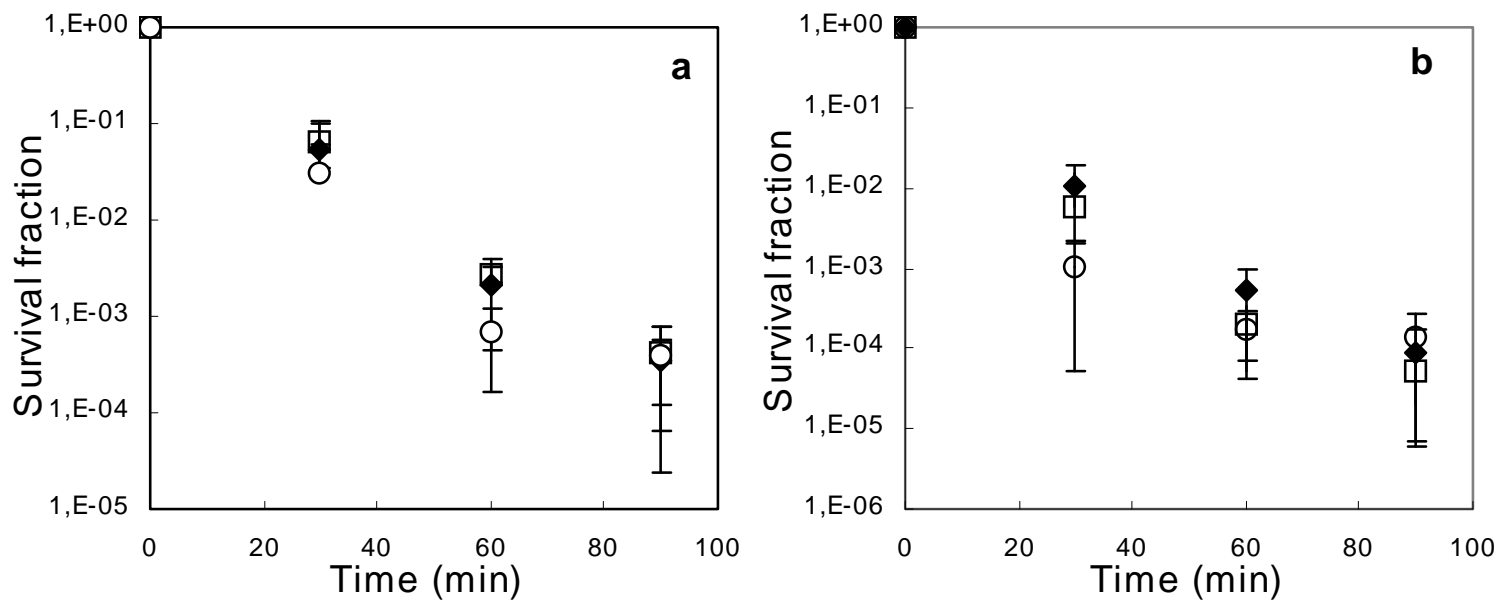

\section{Experimental Section}

\subsection{Bacterial Strains and Media}

E. coli AG100 (K-12 argE3 thi-1 rpsL xyl mtl $\Delta$ (gal-uvrB) supE44) [27], AG112 (marR 5-bp deletion) [28], AG100A ( $\Delta(\operatorname{acr} A B):: K a n)$ [19], AG102K (marR $\Delta(\operatorname{acr} A B):: K a n)$ [19] and AG100/Kan $(\triangle(\operatorname{marCORAB}):: \operatorname{Kan})[29]$ were all kindly donated by Dr. S. B. Levy, Center for Adaptation Genetics and Drug Resistance, Tufts University School of Medicine, Boston MA, USA. All strains were maintained on Luria-Bertani (LB) Agar (10 g Tryptone (Merck), 5 g Yeast extract (Merck), 5 g NaCl, $12 \mathrm{~g}$ agar (Difco) and $10 \mathrm{~mL} \mathrm{10 \%} \mathrm{(w/v)} \mathrm{D-glucose,} \mathrm{separately} \mathrm{autoclaved} \mathrm{l}^{-1}$ ); and where appropriate supplemented with $12.5 \mu \mathrm{g}$ kanamycin $\mathrm{mL}^{-1}$.

\subsection{Solvent Killing Assay}

Overnight cultures of E. coli in modified LB Broth (10 g Tryptone (Merck), $5 \mathrm{~g}$ Yeast extract (Merck) and $10 \mathrm{~mL} 10 \%(\mathrm{w} / \mathrm{v})$ D-glucose, separately autoclaved $\left.1^{-1}\right)$ were re-inoculated $(1: 100)$ into the same medium with or without sodium salicylate $(2 \mathrm{mM})$ and shaken vigorously at $37^{\circ} \mathrm{C}$. In mid- to late exponential phase $\left(\approx 5 \times 10^{8} \mathrm{cfu} \mathrm{mL}^{-1} ; 3-4 \mathrm{~h}\right)$, 1-propanol $(4.05 \%$, w/w) or ethanol $(10.0 \%, \mathrm{w} / \mathrm{w})$ was added. Shaking was continued at $37{ }^{\circ} \mathrm{C}$ with samples being removed every $30 \mathrm{~min}$ for subsequent dilution in cold Dil (1 $\mathrm{g}$ Nutrient Broth (Merck), $5 \mathrm{~g} \mathrm{NaCl} \mathrm{L}^{-1}$ ) before plating on LB Agar. Plates were incubated overnight at $37^{\circ} \mathrm{C}$ for the determination of viable counts. Survival at each time point was calculated as fractions of the viable count at the time of solvent addition. Data were statistically treated using the unpaired t-test. Optical density of liquid cultures was measured at $635 \mathrm{~nm}$ using an Aquanalplus Spectro $^{\text {TM }}$ water analyser (Riedel-de-Haën, Germany). 


\section{Conclusions}

In this study we have investigated the influence of small water miscible alcohols ethanol and 1-propanol on a Mar constitutive mutant and a mar deletion mutant of E. coli K-12, as well as the corresponding strains carrying the additional acrAB deletion. In contrast to hydrophobic solvents, all strains were killed in exponential phase by 1-propanol and ethanol at rates comparable to the parent strain. Although the inducible Mar phenotype of Escherichia coli is associated with increased tolerance to multiple hydrophobic antibiotics as well as some highly hydrophobic organic solvents such as cyclohexane, mediated mainly through the AcrAB/TolC efflux system, the Mar phenotype does not appear to protect E. coli from killing by these more polar solvents. Interestingly, sodium salicylate, at concentrations known to induce the mar operon, was unable to increase 1-propanol or ethanol tolerance. Rather, the toxicity of both solvents was increased in the presence of sodium salicylate. Collectively, the results imply that the resilience of E. coli to water miscible alcohols, in contrast to more hydrophobic solvents, does not depend upon the AcrAB/TolC efflux system. Importantly, these results help define the structural limits, in terms of molecular size and functionality, necessary for AcrAB/TolC efflux system substrates.

Furthermore, the inherent stability of microbiological systems, both phage [41] and host cells, towards media containing high contents of organic solvents is of importance when using phage display strategies for screening for recognition motifs selective for relatively hydrophobic targets of nonbiological origin [42]. Moreover, the establishment of phage display protocols suitable for use in media of low dielectricity [43], requires host cell systems capable of withstanding solvent-induced stress. Again, the results presented should assist in the development of such protocols.

\section{Acknowledgements}

The authors acknowledge the Swedish Research Council (VR), Graninge Foundation and Linnaeus University for financial support, and thank Britt-Inger Marklund for constructive discussion.

\section{References and Notes}

1. Vermuë, M.; Sikkema, J.; Verheul, A.; Bakker, R.; Tramper, J. Toxicity of homologous series of organic solvents for the Gram-positive bacteria Arthrobacter and Nocardia sp. and the Gramnegative bacteria Acinetobacter and Pseudomonas sp. Biotechnol. Bioeng. 1993, 42, 747-758.

2. Ingram, L.O. Mechanism of lysis of Escherichia coli by ethanol and other chaotropic agents. $J$. Bacteriol. 1981, 146, 331-336.

3. Albert, A. Selective Toxicity, 7th ed.; Chapman and Hall: London, UK, 1985.

4. Sikkema, J.; Poolman, B.; Konings, W.N.; de Bont, J.A. Effects of the membrane action of tetralin on the functional and structural properties of artificial and bacterial membranes. J. Bacteriol. 1992, 174, 2986-2992.

5. Sikkema, J.; de Bont, J.A.; Poolman, B. Interactions of cyclic hydrocarbons with biological membranes. J. Biol. Chem. 1994, 269, 8022-8028. 
6. Cartwright, C.P.; Veazey, F.J.; Rose, A.H. Effect of ethanol on activity of the plasma-membrane ATPase in, and accumulation of glycine by Saccharomyces cerevisiae. J. Gen. Microbiol. 1987, 133, 857-865.

7. Eaton, L.C.; Tedder, T.F.; Ingram, L.O. Effects of fatty acid composition on the sensitivity of membrane functions to ethanol in Escherichia coli. Subst. Alcohol Actions Misuse 1982, 3, 77-87.

8. Cohen, S.P.; Yan, W.; Levy, S.B. A multidrug resistance regulatory chromosomal locus is widespread among enteric bacteria. J. Infect. Dis. 1993, 168, 484-488.

9. McDermott, P.F.; White, D.G.; Podglajen, I.; Alekshun, M.N.; Levy, S.B. Multidrug resistance following expression of the Escherichia coli marA gene in Mycobacterium smegmatis. J. Bacteriol. 1998, 180, 2995-2998.

10. Kunonga, N.I.; Sobieski, R.J.; Crupper, S.S. Prevalence of the multiple antibiotic resistance operon (marRAB) in the genus Salmonella. FEMS Microbiol. Lett. 2000, 187, 155-160.

11. Cohen, S.P.; Hachler, H.; Levy, S.B. Genetic and functional analysis of the multiple antibiotic resistance (mar) locus in Escherichia coli. J. Bacteriol. 1993, 175, 1484-1492.

12. Barbosa, T.M.; Levy, S.B. Differential expression of over 60 chromosomal genes in Escherichia coli by constitutive expression of MarA. J. Bacteriol. 2000, 182, 3467-3474.

13. Ma, D.; Cook, D.N.; Alberti, M.; Pon, N.G.; Nikaido, H; Hearst, J.E. Molecular cloning and characterization of acrA and acrE genes of Escherichia coli. J. Bacteriol. 1993, 175, 6299-6313.

14. Ma, D.; Alberti, M.; Lynch, C.; Nikaido, H.; Hearst, J.E. The local repressor AcrR plays a modulating role in the regulation of acrAB genes of Escherichia coli by global stress signals. Mol. Microbiol. 1996, 19, 101-112.

15. Thanassi, D.G.; Cheng, L.W.; Nikaido, H. Active efflux of bile salts by Escherichia coli. J. Bacteriol. 1997, 179, 2512-2518.

16. Moken, M.C.; McMurry, L.M.; Levy, S.B. Selection of multiple-antibiotic-resistant (mar) mutants of Escherichia coli by using the disinfectant pine oil: roles of the mar and acrAB loci. Antimicrob. Agents Chemother. 1997, 41, 2770-2772.

17. McMurry, L.M.; Oethinger, M.; Levy, S.B. Overexpression of marA, soxS, or acrAB produces resistance to triclosan in laboratory and clinical strains of Escherichia coli. FEMS Microbiol. Lett. 1998, 166, 305-309.

18. Hachler, H.; Cohen, S.P.; Levy, S.B. marA, a regulated locus which controls expression of chromosomal multiple antibiotic resistance in Escherichia coli. J. Bacteriol. 1991, 173, 5532-5538.

19. Okusu, H.; Ma, D.; Nikaido, H. AcrAB efflux pump plays a major role in the antibiotic resistance phenotype of Escherichia coli multiple-antibiotic-resistance (Mar) mutants. J. Bacteriol. 1996, 178, 306-308.

20. Martin, R.G.; Rosner, J.L. Binding of purified multiple antibiotic-resistance repressor protein (MarR) to mar operator sequences. Proc. Natl. Acad. Sci. USA 1995, 92, 5456-5460.

21. Alekshun, M.N.; Levy, S.B. Alteration of the repressor activity of MarR, the negative regulator of the Escherichia coli marRAB locus, by multiple chemicals in vitro. J. Bacteriol. 1999, 181, 4669-4672. 
22. Cohen, S.P.; Levy, S.B.; Foulds, J.; Rosner, J.L. Salicylate induction of antibiotic resistance in Escherichia coli: Activation of the mar operon and a mar-independent pathway. J. Bacteriol. 1993, 175, 7856-7862.

23. Brooun, A.; Tomashek, J.J.; Lewis, K. Purification and ligand binding of EmrR, a regulator of a multidrug transporter. J. Bacteriol. 1999, 181, 5131-5133.

24. Aono, R.; Kobayashi, M.; Nakajima, H.; Kobayashi, H. A close correlation between improvement of organic solvent tolerance levels and alteration of resistance toward low levels of multiple antibiotics in Escherichia coli. Biosci. Biotechnol. Biochem. 1995, 59, 213-218.

25. Asako, H.; Nakajima, H.; Kobayashi, K.; Kobayashi, M.; Aono, R. Organic solvent tolerance and antibiotic resistance increased by overexpression of marA in Escherichia coli. Appl. Environ. Microbiol. 1997, 63, 1428-1433.

26. Oethinger, M.; Kern, W.V.; Goldman, J.D.; Levy, S.B. Association of organic solvent tolerance and fluoroquinolone resistance in clinical isolates of Escherichia coli. J. Antimicrob. Chemother. 1998, 41, 111-114.

27. George, A.M.; Levy, S.B. Amplifiable resistance to tetracycline, chloramphenicol, and other antibiotics in Escherichia coli: involvement of a non-plasmid-determined efflux of tetracycline. $J$. Bacteriol. 1983, 155, 531-540.

28. Oethinger, M.; Kern, W.V.; Jellen-Ritter, A.S.; McMurry, L.M.; Levy, S.B. Ineffectiveness of topoisomerase mutations in mediating clinically significant fluoroquinolone resistance in Escherichia coli in the absence of the AcrAB efflux pump. Antimicrob. Agents Chemother. 2000, 44, 10-13.

29. Maneewannakul, K.; Levy, S.B. Identification for mar mutants among quinolone-resistant clinical isolates of Escherichia coli. Antimicrob. Agents Chemother. 1996, 40, 1695-1698.

30. Farewell, A.; Kvint, K.; Nystrom, T. uspB, a new sigmaS-regulated gene in Escherichia coli which is required for stationary-phase resistance to ethanol. J. Bacteriol. 1998, 180, 6140-6147.

31. Fried, V.A.; Novick, A. Organic solvents as probes for the structure and function of the bacterial membrane: effects of ethanol on the wild type and an ethanol- resistant mutant of Escherichia coli K-12. J. Bacteriol. 1973, 114, 239-248.

32. Ingram, L.O.; Vreeland, N.S. Differential effects of ethanol and hexanol on the Escherichia coli cell envelope. J. Bacteriol. 1980, 144, 481-488.

33. Jordan, S.L.; Glover, J.; Malcolm, L.; Thomson-Carter, F.M.; Booth, I.R.; Park, S.F. Augmentation of killing of Escherichia coli O157 by combinations of lactate, ethanol, and lowpH conditions. Appl. Environ. Microbiol. 1999, 65, 1308-1311.

34. Aumercier, M.; Murray, D.M.; Rosner, J.L. Potentiation of susceptibility to aminoglycosides by salicylate in Escherichia coli. Antimicrob. Agents Chemother. 1990, 34, 786-791.

35. Domenico, P.; Hopkins, T.; Schoch, P.E.; Cunha, B.A. Potentiation of aminoglycoside inhibition and reduction of capsular polysaccharide production in Klebsiella pneumoniae by sodium salicylate. J. Antimicrob. Chemother. 1990, 25, 903-914.

36. Damper, P.D.; Epstein, W. Role of the membrane potential in bacterial resistance to aminoglycoside antibiotics. Antimicrob. Agents Chemother. 1981, 20, 803-808. 
37. Bryan, L.E.; Kwan, S. Roles of ribosomal binding, membrane potential, and electron transport in bacterial uptake of streptomycin and gentamicin. Antimicrob. Agents Chemother. 1983, 23, 835-845.

38. Tsukagoshi, N.; Aono, R. Entry into and release of solvents by Escherichia coli in an organicaqueous two-liquid-phase system and substrate specificity of the AcrAB-TolC solvent-extruding pump. J. Bacteriol. 2000, 182, 4803-4810.

39. Ferhat, M.; Atlan, D.; Vianney, A.; Lazzaroni, J.C.; Doublet, P.; Gilbert, C. The TolC protein of Legionella pneumophila plays a major role in multi-drug resistance and the early steps of host invasion. PLOS one 2009, 4, e7732.

40. Ricci, V.; Piddock, L.J.V. Only for substrate antibiotics are a functional AcrAB-TolC efflux pump and RamA required to select multidrug-resistant Salmonella Typhimurium. J. Antimicrob. Chemother. 2009, 63, 654-657.

41. Olofsson, L.; Ankarloo, J.; Andersson, P.O.; Nicholls, I.A. Bacteriophage viability in organic solvents. Chem. Biol. 2001, 8, 661-671.

42. Berglund, J.; Nicholls, I.A.; Lindbladh, C.; Mosbach, K. Selection of phage display peptides with affinity for a yohimbine imprinted methacrylate polymer. Anal. Commun. 1998, 35, 3-7.

43. Söderberg, P.; Olofsson, L.; Ankarloo, J.; Nicholls, I.A. Phage display screening in low dielectric media. J. Mol. Recognit. 2008, 29, 329-336.

(C) 2010 by the authors; licensee Molecular Diversity Preservation International, Basel, Switzerland. This article is an open-access article distributed under the terms and conditions of the Creative Commons Attribution license (http://creativecommons.org/licenses/by/3.0/). 